\title{
黄酩醇类化合物的合成研究进展
}

\author{
梅青刚 $a, b$ 袁伟成 ${ }^{a}$ 王 凉*, $b$ \\ $\left({ }^{a}\right.$ 中国科学院成都有机化学研究所 成都 610041) \\ ( ${ }^{b}$ 中国科学院成都生物研究所 成都 610041)
}

\begin{abstract}
摘要 黄酮醇即 3-羟基黄酮, 是一类独特的黄酮化合物, 广泛存在于植物界中, 具有许多重要的生物活性和药理作用, 其化学合成研究一直备受关注. 综述了黄酮醇骨架的 4 种主要合成方法: Auwers 法, 查尔酮氧化关环即 Algar-FlynnOyamada (AFO)反应, Baker-Venkataraman 法, 黄酮 DMDO (3,3-二甲基双环氧乙烷)氧化法; 并对多着基黄酮醇的区域 选择性甲基化、异戌烯基化和糖苷化等衍生化方法进行了概述.
\end{abstract}

关键词 黄酮醇; 合成; AFO 反应; 衍生化; 区域选择性

\section{Progress in the Synthesis of 3-Hydroxyflavones}

\author{
Mei, Qinggang ${ }^{a, b} \quad$ Yuan, Weicheng $^{a} \quad$ Wang, Chun ${ }^{*, b}$ \\ ( ${ }^{a}$ Chengdu Institute of Organic Chemistry, Chinese Academy of Sciences, Chengdu 610041) \\ ( ${ }^{b}$ Chengdu Institute of Biology, Chinese Academy of Sciences, Chengdu 610041)
}

\begin{abstract}
Flavonols, also known as 3-hydroxyflavones, are a kind of unique flavonoids that widely distributed in the plant kingdom, and have been associated with a wide variety of biological activities and pharmaceutical applications. Four principal synthetic approaches to flavonol skeleton including Auwers synthesis, Algar-Flynn-Oyamada reaction (AFO), Baker-Venkataraman synthesis and flavone oxidation by 3,3-dimethyldioxirane (DMDO) are summarized. The regioselective methylation, prenylation and glycosylation of polyhydroxyl flavonols are presented as well.
\end{abstract}

Keywords flavonol; synthesis; AFO reaction; derivatization; regioselectivity

黄酮醇即 3-羊基黄酮，是一类独特的黄酮化合物， 广泛存在于植物界中, 数量众多, 结构复杂多样, 具有 许多重要的生物活性和药理作用. 它们不仅能够清除人 体内的自由基, 具备抗氧化功效; 还能解毒抗炎, 阻断 血小板的凝集, 抑制癌症的发生, 对人类的许多疾病具 有治疗作用; 而且, 还能增强心脑血管血流量, 调节代 谢, 提高人体免疫力 ${ }^{[1 ~ 3]}$. 黄酮醇的药用价值已为人们 所熟知, 西兰花、西红柿、洋苟和苹果等蔬菜水果, 茶 叶和红酒等饮品都富含黄酮醇, 经常食用有助于降低冠 心病的发病率 ${ }^{[4]}$.

在植物的生长繁殖过程中, 黄酮醇同样扮演着重要 角色. 黄酩醇类化合物使植物呈现各种花色, 吸引传粉 者, 保护植物免受紫外光、昆虫、细菌、真菌和病毒的 伤害, 还作为植物激素的调控者. 玉米和矮牵牛花的花 粉突变体, 因缺少了黄酮醇而不能发芽; 然而, 倘若在
授粉时添加山柰酚，这种缺陷就能予以弥补，突变体花 粉仍能正常生长发育 ${ }^{[5]}$.

此外，黄酮醇及其衍生物还是一类典型的具有激发 态分子内质子转移(ESIPT) 特性的荧光化合物, 互变异 构诱导产生双荧光现象, 其荧光行为对分子环境诸如极 性、氢键作用等非常敏感, 被誉为多参数、多功能的第 二代苂光探针 ${ }^{[6]}$. 作为一种溶剂变色染料, 有望制成分 子苂光传感器, 用于监测生物分子间的反应 ${ }^{[7,8]}$. 近年研 究发现, 用噻吩作为 $\mathrm{B}$ 环合成的黄酮醇类似物, 可作为 散装异质结太阳能电池的一种材料 ${ }^{[9]}$.

黄酮醇这些独特的生物活性和优良的物理化学性 质, 引起了研究者的广泛兴趣, 其化学合成方法研究日 益受到重视. 本文就近年来黄酮醇及其衍生物的合成研 究作一综述.

* E-mail: wangchun@cib.ac.cn

Received August 29, 2014; revised September 12, 2014; published online September 16, 2014.

Project supported by the National Natural Science Foundation of China (No. 21172214).

国家自然科学基金(No. 21172214)资助项目. 


\section{1 黄酮醇骨架的合成}

黄酮醇骨架的合成, 关键点不仅仅在于苯并 $\gamma$-吡喃 酮环的形成, 还在于 3-差基的引入. 这两个步骤可以合 二为一，同时完成，如: Auwers 法、查尔酮氧化关环即 AFO 反应; 也可以分步进行, 即先合成黄酮, 再引入羟 基, 如: Baker-Venkataraman 法、黄酮 DMDO 氧化法.

\subsection{Auwers 合成法}

Auwers 法 ${ }^{[10,11]}$ 是较早的黄酮醇合成法, 由 Auwers 于 1908 年发现. 苯并呋喃与苯甲醛缩合, 溴化生成 2溴-2-( $\alpha$-溴苠基)苯并呋喃酮, 然后经醇碱处理发生重排, 转变为黄酮醇(Scheme 1).

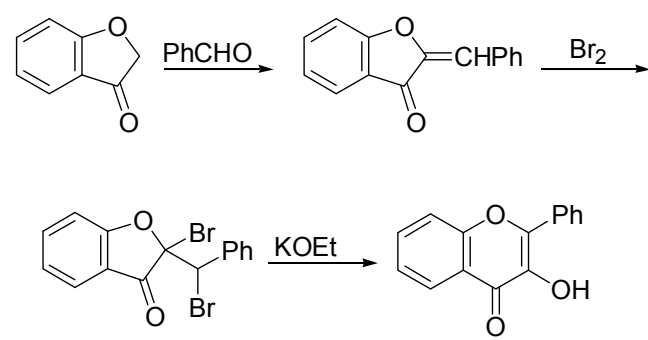

图式 1 Auwers 法合成黄酮醇

Scheme 1 Auwers synthesis of flavonol

由于该反应的底物适用面较窄, 中间体二溴代物制 备较难, 反应步骤较多, 产率偏低, 因此在有机合成中 的实际应用较少.

\subsection{Algar-Flynn-Oyamada (AFO)反应}

查耳酮氧化关环即 Algar-Flynn-Oyamada (AFO)反 应, 最初由爱尔兰的 Algar, Flynn 和日本的 Oyamada 在 1934 年同时报道, 后经 Ozawa 及 Smith 等的改进, 成为 制备黄酮醇类化合物的经典方法, 被广泛运用于各类黄<smiles>[R]COc1cc(C=O)cc(Oc2ccc(C(=O)CCC(O)C(C)C)c(O)c2)c1</smiles>

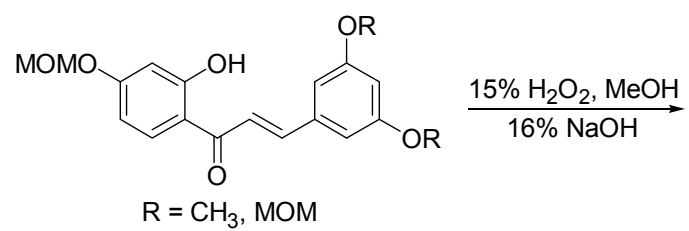<smiles></smiles><smiles>[R2]Oc1cc(C=[R])cc(-c2oc3cc(O)ccc3c(=O)c2O)c1</smiles>

图式 2 汪小组 $\mathrm{AFO}$ 反应合成黄酮醇的路线

Scheme 2 Synthetic route of flavonols via AFO reaction used by Wang 


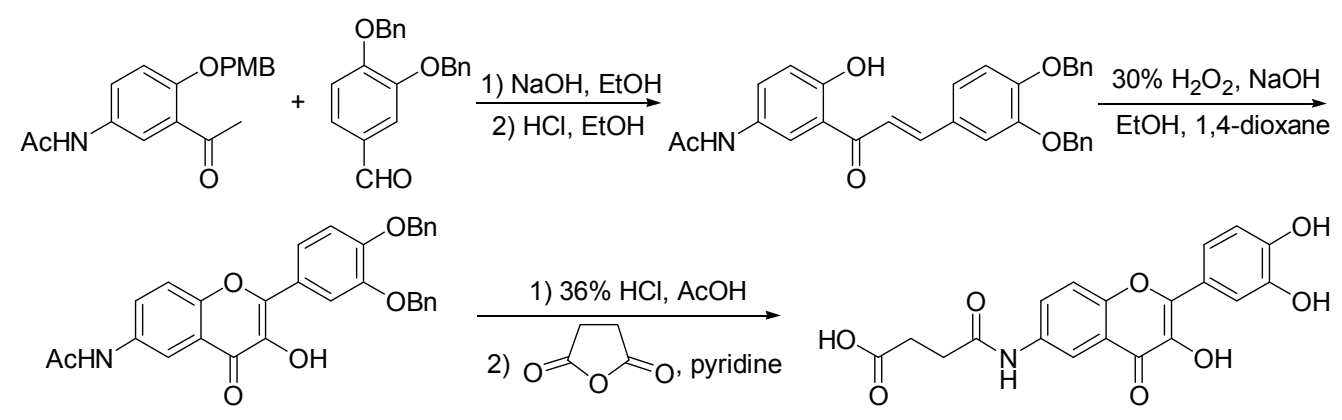

图式 3 Yap 小组水溶性黄酮醇的合成路线

Scheme 3 Synthetic route of water-soluble flavonols used by Yap

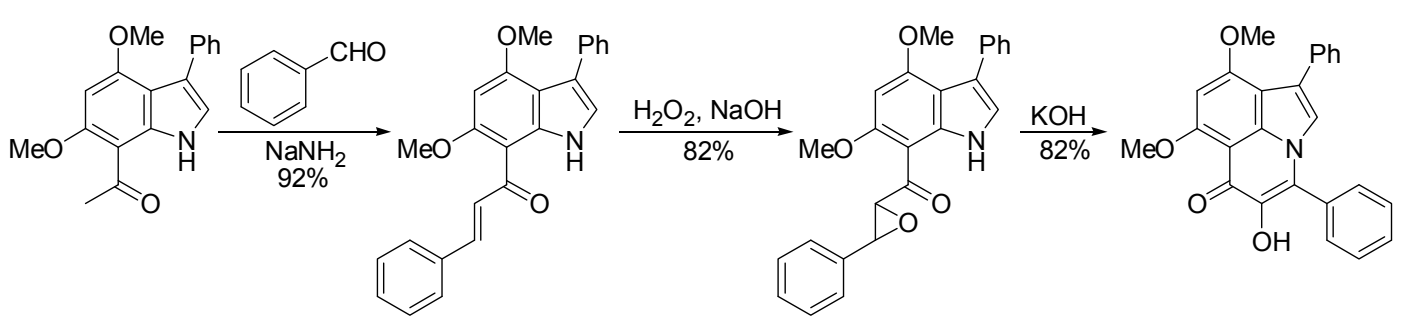

图式 4 吲哚黄酮醇类似物的合成路线

Scheme 4 Synthetic route of indole analogues of flavonols

药耐药性射流百功能, 故而能加强青蒿素的抗疮活性以 及诺氟沙星的抑菌活性 ${ }^{[17]}$. 虽然后来在多种植物中均 有发现, 但天然丰度都较低. 2008 年, Kraus 科研小组 ${ }^{[18]}$ 实现了其化学合成, 以三甲氧基邻羟基苯乙酮为原料, 与二苄氧基苯甲醛羟醛缩合先制得查尔酮. 在随后的 $\mathrm{AFO}$ 反应中, 由于邻位甲氧基的存在, 他们使用了过量 的 $\mathrm{H}_{2} \mathrm{O}_{2}$ 和 $\mathrm{NaOH}$, 并延长了反应时间, 先以 $60 \%$ 的产率 制得了二氢黄酩醇, 再用 $\mathrm{I}_{2}$ 氧化, 以 $78 \%$ 的产率制得了 黄酮醇, 完成 $\mathrm{AFO}$ 氧化环合反应. 最终, 以 $31 \%$ 的总产 率实现了 Chrysosplenol D 的人工合成(Scheme 5). 这种 先制得二氢黄酮醇再用 $I_{2}$ 氧化脱氢的方法, 将传统的 AFO 反应分为两步进行, 产率适中, 中间体二氢黄酮醇 易于纯化咜存, 这对于 AFO 反应的深入研究具有一定 指导意义.

2010 年, Lewin 课题组 ${ }^{[19]}$ 以甜橙中含量丰富的橙皮 苷为原料，运用改进的 $\mathrm{AFO}$ 法，分 2 次加入 $30 \%$ 的 $\mathrm{H}_{2} \mathrm{O}_{2}$ 溶液, 低温下反应 $2 \mathrm{~d}$, 然后用乙酸调节体系的 $\mathrm{pH}$ 值至 6, 再加入焦亚硫酸钠回流 $2 \mathrm{~h}$, 最后在酸性条件下脱糖, “一锅” 内合成了 4'-甲基榆皮素(Scheme 6). 这种改进 将氧化、酸化和脱氢在 “一锅” 内连续进行, 操作简便 易行, 适合于大量的制备; 但反应时间长, 产率较低, 脱糖后收率仅为 $14 \%$.

Pandurangan 等 ${ }^{[20]}$ 结合上述两种改进方法, 用 $\mathrm{H}_{2} \mathrm{O}_{2} / \mathrm{NaOH}$ 氧化得到查尔酮环氧化物, 该环氧化物在 酸性条件下选择性开环生成二氢黄酮醇, 再加入焦亚硫 酸钾溶液回流, 以中等收率合成了黄酮醇类化合物
(Scheme 7). 该方法选用 MOM 作保护基，得到了 AFO 反应的两个关键中间体, MOM 保护基在酸化生成二氢 黄酮醇时一并脱除; 而且, 选用焦亚硫酸钾氧化脱氢, 后处理方便，避免了 $\mathrm{I}_{2}$ 氧化的繁琐步骤，尽管产率有所 下降，但对于多羟基黄酮醇的合成，不失为一种较好的 改进方法.

2012 年, Gunduz 等 ${ }^{[2]}$ 对经典的 AFO 反应进一步进 行了改进, 发展了 “一锅法” $\mathrm{AFO}$ 环合法. 以邻羟基苯 乙酮与取代苯甲醛为原料, 在氢氧化钠甲醇溶液中回流 反应 $3 \mathrm{~h}$, 冷却至室温后, 直接加入 $0.5 \mathrm{~mol} \cdot \mathrm{L}^{-1}$ 的氢氧 化钠和 $35 \%$ 的 $\mathrm{H}_{2} \mathrm{O}_{2}$ 溶液搅拌 2 3 h, “一锅” 内连续操 作合成了黄酮醇及其类似物 (噻吩取代的 3-羟基黄酮) (Scheme 8). 反应没有查尔酮和橙酮等副产物生成, 粗 产物只需用 EtOAc 萃取或者简单的过滤处理, 就可以得 到目标产物。该方法显著的特点是: 利用甲酰基苯硼酸 “一锅” 操作合成了 4'-差基黄酮醇, 而用其它方法很 难一步完成; 简化了查尔酮中间体的制备步骤, 合成时 间大大缩短，分离纯化简单易行，产率明显提高，最高 可达 $79 \%$, 值得进一步研究完善.

人们对 $\mathrm{AFO}$ 反应的机理进行了广泛的研究 ${ }^{[22 ~ 24]}$, 下面是 Geismann 等 $^{[25]}$ 提出的一种可能的反应机理 (Scheme 9): 查尔酮在过氧化氢作用下转化为环氧化物 中间体，然后酚氧负离子对环氧化物进行亲核进攻，环 化为二氢黄酮醇, 继而被氧化为黄酮醇. 若环氧化物中 的酚氧负离子对羰基 $\beta$-位进行进攻, 则生成二氢黄酮 醇; 进攻羰基 $\alpha$-位, 则生成副产物五元环的橙酮. 


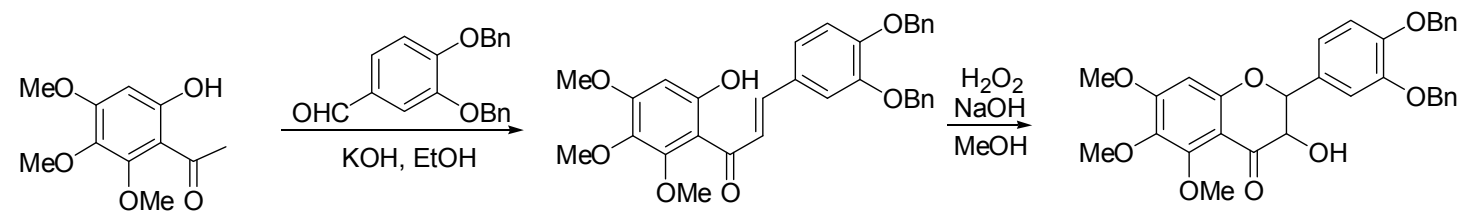

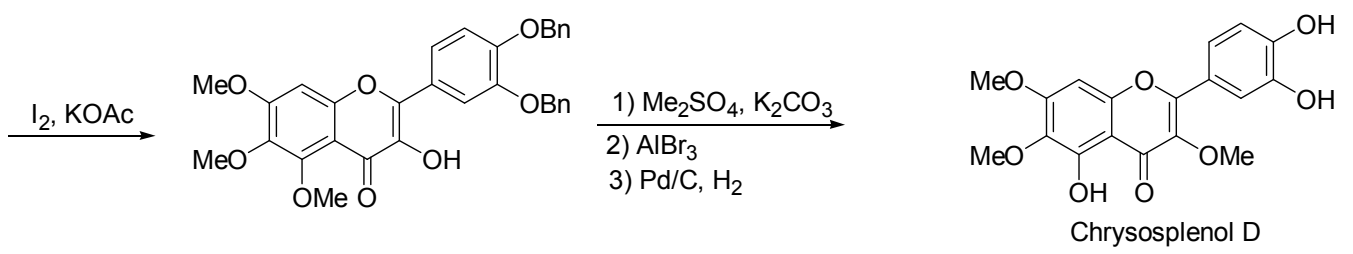

图式 5 Chrysosplenol D 的合成路线

Scheme 5 Synthetic route of chrysosplenol D

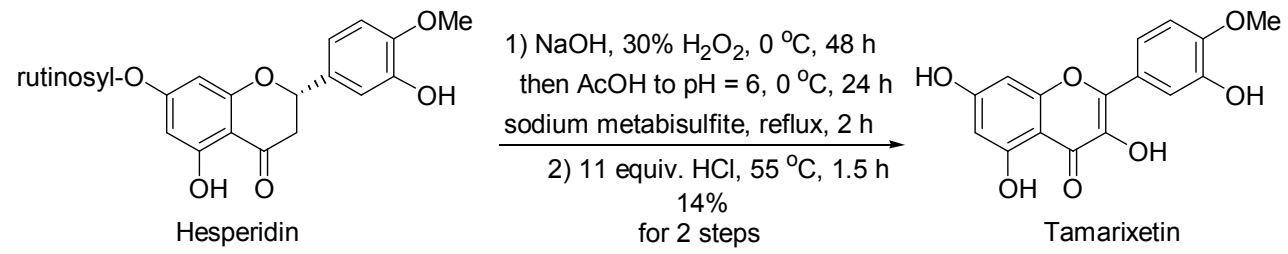

图式 6 由橙皮苷合成 4'-甲基檞皮素

Scheme 6 Synthesis of tamarixetin from hesperidin<smiles>[R]c1ccc(CC(C(=O)c2cc(OC)cc(OC)c2C(=O)Cc2cc(-c3oc4cc(O)cc(O)c4c(=O)c3-c3ccc([R])c([R])c3)ccc2[R])c2cc(OC)cc(OC)c2OC)cc1[R]</smiles>

图式 7 Pandurangan 等多羟基黄酮醇的合成路线

Scheme 7 Synthetic route of polyhydroxyl flavonols used by Pandurangan

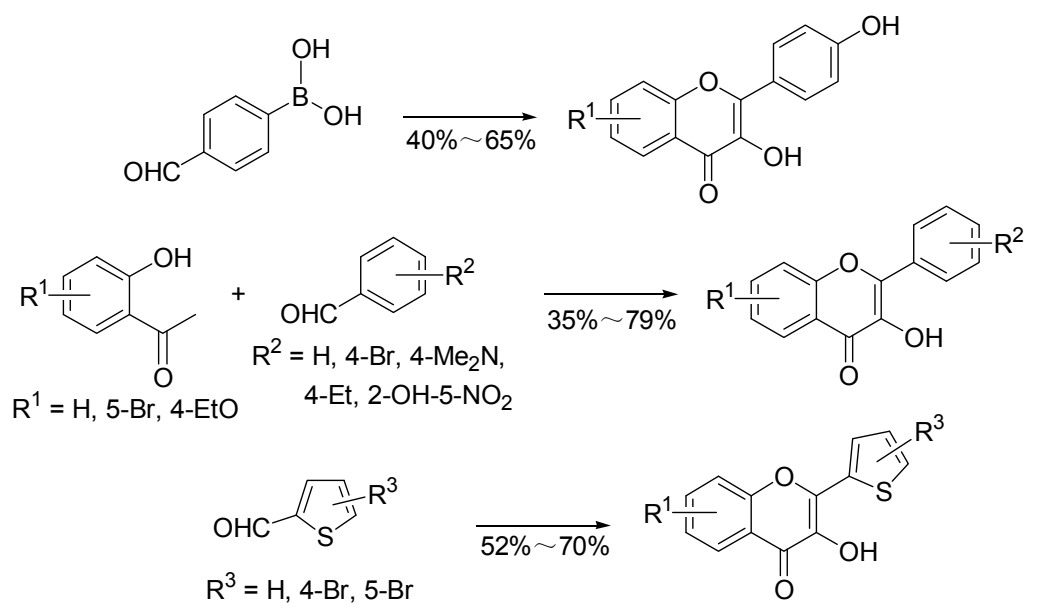

图式 8 Gunduz 等的黄酮醇 “一锅” 合成法

Scheme 8 "One-pot" synthesis of flavonols used by Gunduz 


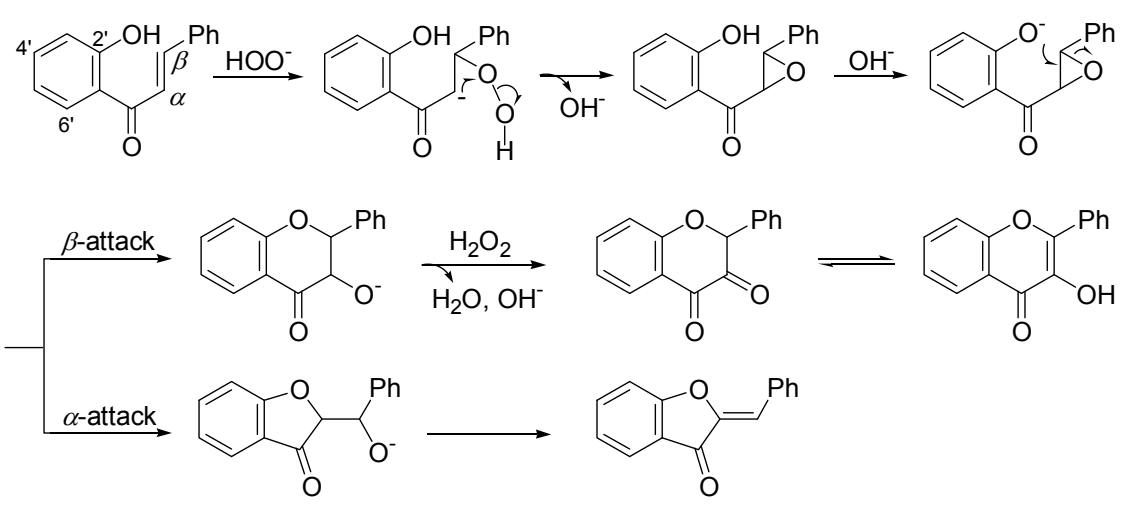

图式 9 AFO 反应合成黄酮醇的可能机理

Scheme 9 Possible mechanism of AFO reaction

反应机理能够解释许多实验现象. 例如, 对于 6'-位 没有取代基或者取代基体积较小的查尔酮，应用 $\mathrm{AFO}$ 反应得到黄酩醇类化合物的收率较高(Schemes 2 和 5); 而当 6'-位取代基体积过大时, 其对羰基 $(\mathrm{C}=\mathrm{O})$ 的立体 位阻作用致使 2'-酚羟基与羰基 $\beta$-位的距离增大，与 $\alpha$ 位的距离拉近, 因此, 副产物五元环橙酮的量增加 ${ }^{[26]}$, 而黄酩醇的收率明显下降(Scheme 7).

然而, 该反应机理也备受质疑一一是否形成环氧化 物中间体 ${ }^{[22,23]}$. 在上述条件下, 2 '-差基查尔酮环氧化物 中间体一直未能分离得到 ${ }^{[15]}$; 但是, 当 $2^{\prime}$-羟基被 OMOM、甲氧基等取代时, 在同样条件下, 分离得到唯 一的产物查尔酮环氧化物(Scheme 7).

$\mathrm{AFO}$ 反应, 从发现至今已有 80 年的历史, 仍然是 目前人们普遍采用的一种黄酮醇合成法. 其廉价易得的 底物原料, 普通寻常的反应试剂, 简单易行的操作步骤, 是其它方法难以企及的. 但是, 反应产率受底物及条件 的影响较大, 规律性还不够明朗, 反应有橙酮、二氢黄 酮醇、2-芐基-2-羟基二氢苯并呋喃-3-酮以及 2-芳基苯并 呋喃-3-羧酸等副产物生成, 在大规模生产和应用上仍 有一定的局限性. 人们正围绕 $\mathrm{AFO}$ 反应的机理研究, 分 离纯化可能的中间体, 探索影响反应的因素, 以期减少 副反应的发生，从而最终提高 $\mathrm{AFO}$ 反应的产率.

\subsection{Baker-Venkataraman 法}

由 Allan-Robinson 反应演变而来的 Baker-Venkataraman 法 ${ }^{[27,28]}$, 是通用的黄酮类化合物合成方法. 包括 3 个步骤: 邻羟基苯乙酮与芳甲酰氯(芳甲酸酐或芳甲 酸)在吡啶中生成酯; 然后, 在碱性条件下发生 Baker-Venkataraman 重排反应, 得到 $\beta$-丙二酮化合物, 经酸催化闭环生成黄酮类化合物(Scheme 10).

当 $\mathrm{R}$ 基团为烷基(通常为甲基或芐基)或苯甲酰基 时，闭环生成的 3- $O$-位取代黄酮脱去烷基或苯甲酰基, 就得到了黄酮醇化合物. 苯甲酰氧基的导入, 可以通过 引入溴原子间接进行 ${ }^{[29]}$ (Scheme 11).

人们对 Baker-Venkataraman 法的改进, 多集中于使 用不同的碱催化体系(如碳酸钾、叔丁醇钾、有机锂试 剂), 硅烷基羟基保护以及相转移催化法等, 将邻羟基 苯乙酮直接转化为 $\beta$-丙二酮, 使得酯化和重排两步反应 能够一步进行.

更多的研究者尝试将重排和环合两步合二为一. Tanaka 等 ${ }^{[30]}$ 在合成一种对花粉的黄酮醇半乳糖基转移 酶(F3GalTase)的形成具有光催化作用的山柰酚衍生物 时, 先将邻羟基苯乙酮与芳香甲酸在 DMAP 等催化下 形成酯; 然后在碳酸钾/吡啶体系中, 酯直接脱水闭环生 成 3-芐氧基黄酮; 最后脱去芐基, 得到黄酮醇化合物 (Scheme 12).

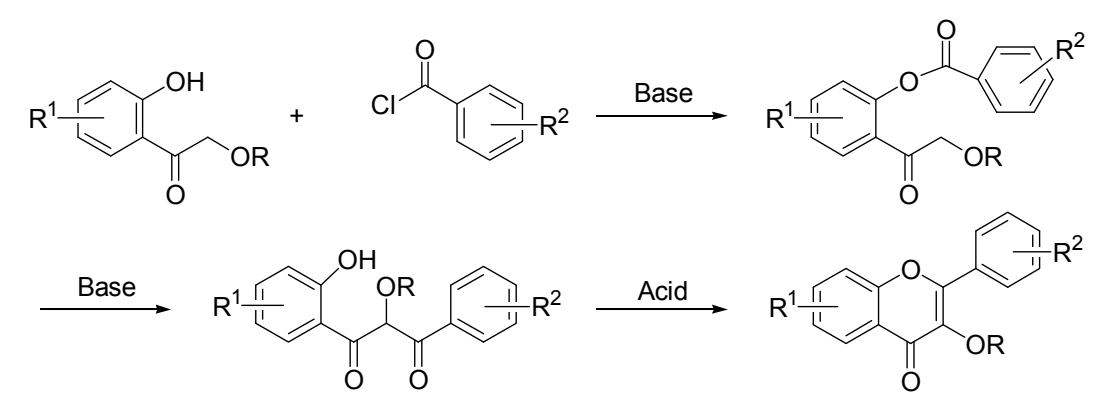

图式 10 Baker-Venkataraman 法合成黄酮的路线

Scheme 10 Baker-Venkataraman synthesis of flavones 


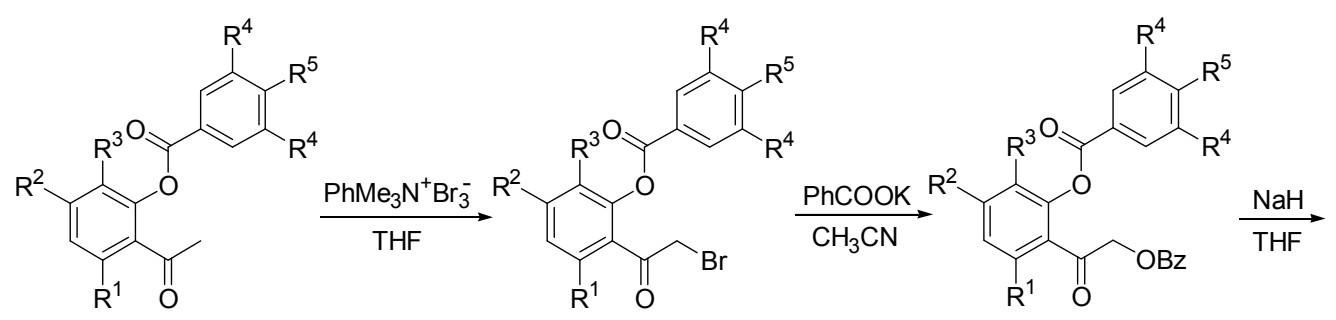

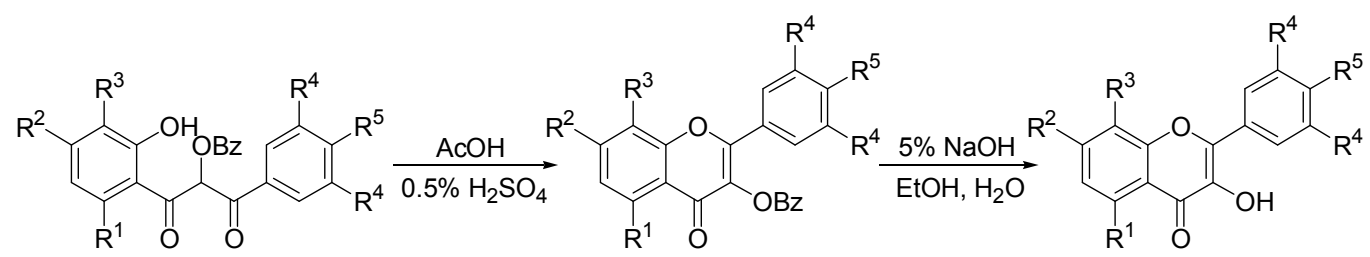

图式 11 Baker-Venkataraman 法合成黄酮醇的路线

Scheme 11 Baker-Venkataraman synthesis of flavonols

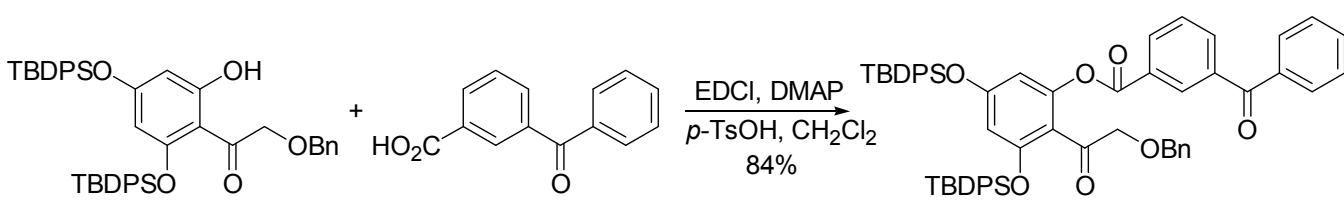

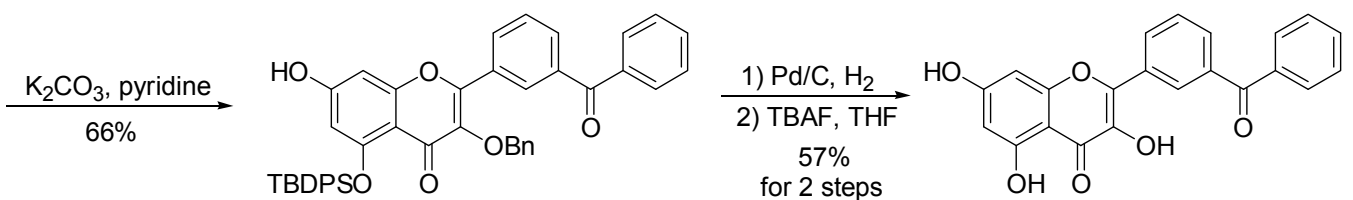

图式 12 Tanaka 小组合成黄酮醇衍生物的路线

Scheme 12 Synthesis route of flavonols used by Tanaka

2007 年, Shaw 课题组 ${ }^{[31]}$ 在合成 4 个不同基团保护的 山柰酚衍生物时, 采用了几乎完全相同的方法. 酯直接 脱水闭环, 产率不高, 仅为 $40 \%$ 左右(Scheme 13). 值得 注意的是, 环合时, 本例中是 5-MOM 保护基脱除; 而 在上例中, 主要得到 7-TBDPS 保护基脱除产物.

Chen 等 ${ }^{[32]}$ 在合成 5,7-二羟基-3- $O-\beta-D$-半乳糖基黄 酮时, 更进一步缩短了反应步骤, 实现了从邻羟基苯乙 酮到黄酩醇的 “一锅法” 合成. 邻羟基苯乙酮与苯甲酸 钠在苯甲酸酐中于 $170{ }^{\circ} \mathrm{C}$ 搅拌 $3 \mathrm{~h}$ 后, 加入氢氧化钾和
乙醇回流 $0.5 \mathrm{~h}$, 之后, 在水溶液中通入 $\mathrm{CO}_{2}$ 气体, 方便 地制得了黄酮醇(Scheme 14). 该合成方法用苯甲酸䣶 作酯化试剂, 生成的酯在碱性条件下脱水闭环, 随后在 水溶液中脱去苯甲酰基, 再用饱和 $\mathrm{CO}_{2}$ 气体酸化; 这种 改进方法将酯化、重排、脱水闭环、苯甲酰基脱除多步 反应合并为两步, 在 “一锅” 内连续操作, 大大简化了 手续，具有时间短、操作简单、溶剂环境友好等优点，同 时产率也有所提高. 不过这种方法, 适应于较简单的底 物, 对于多取代底物, 还需要进一步研究.
MOMO<smiles>COCC(=O)c1c(O)cccc1OC</smiles><smiles>O=C(O)c1ccc(OCc2ccccc2)cc1</smiles><smiles>COCC(=O)c1c(OC)cc(OC)cc1OC(=O)c1ccc(OCc2ccccc2)cc1</smiles><smiles>COc1cc([O-])c2c(=O)c(OC)c(-c3ccc(OCc4ccccc4)cc3)oc2c1</smiles>

图式 13 Shaw 小组合成黄酮醇衍生物的路线

Scheme 13 Synthetic route of flavonols use by Shaw 
<smiles>CCOC(C)C(C)(C)C(C)(C)C(=O)c1ccccc1C(=O)OCC#N</smiles>

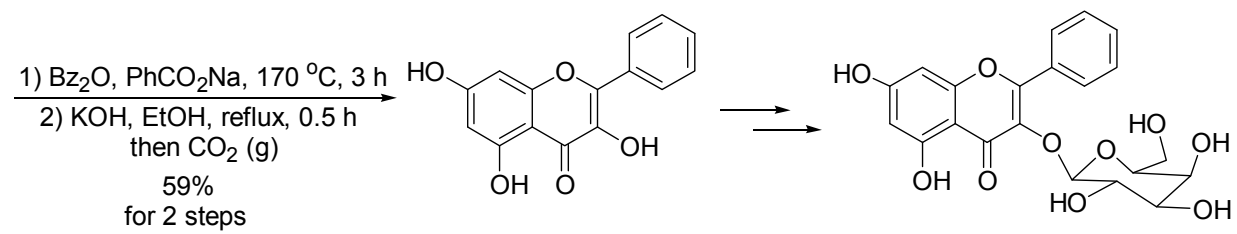

图式 14 Chen 小组合成黄酮醇衍生物的路线

Scheme 14 Synthetic route of flavonols use by Chen

利用 Baker-Venkataraman 法合成黄酮醇类化合物, 需要经过中间体黄酮, 才能得到黄酮醇, 步骤较多, 总 收率也往往不高; 但是, 各步反应易于操作, 中间体的 纯化也易于进行, 更重要的是, 反应底物廉价易得, 人 们还可以根据底物情况, 灵活使用. 因此, 这种传统方 法依然具有良好的应用前景.

\section{4 黄酮 DMDO 氧化}

Costa 等 ${ }^{[33]}$ 早年报道了一种黄酮转化为黄酮醇的方 法. 先用二异丙基氨基锂(LDA)使黄酮的 3-位氢去质子 化生成理盐, 然后用硓酸甲酯处理, 乙酸酸化, $\mathrm{H}_{2} \mathrm{O}_{2}$ 氧 化, 再经水解得到黄酮醇. 该方法黄酮醇的收率较高, 但由于锂盐的制备和砋酸甲酯处理都需要在 $-78{ }^{\circ} \mathrm{C}$ 进 行, 反应条件苛刻, 操作步骤较多, 不适于大量的制备. 人们转而致力于寻找通过氧化黄酮而插入羟基的方法. 但黄酮分子中, 共轭双键由于受到羰基吸电子和烷氧基 供电子的协同作用, 因而, 对于不论是亲电的还是亲核 的氧化试剂, 其均表现为惰性. $\mathrm{H}_{2} \mathrm{O}_{2}$ 曾用来氧化异黄 酮、查尔酮和橙酮, 但是, 用 $m-\mathrm{CPBA}, \mathrm{H}_{2} \mathrm{O}_{2}$ 这类经典的 酸碱性氧化剂以及 $\mathrm{KMnO}_{4}, \mathrm{SeO}_{2}, \mathrm{NiO}_{2}, \mathrm{Tl}(\mathrm{OAc})_{3}$ 等金属 类氧化剂, 对黄酮的直接氧化都未能成功.

二甲基双环氧乙烷(DMDO)是一种温和的烯烃环氧 化试剂. 不论是富电子烯烃, 还是缺电子诸如 $a, \beta$-不饱 和酸、酯、酮等的共轭烯烃, 都能被它环氧化. 1991 年, Adam 科研小组 ${ }^{[34]}$ 首次将 DMDO 应用于黄酮的环氧化, 后来也用于查尔酮和异黄酮的环氧化. 他们采用间歇式 地滴加过量数倍的 DMDO 的丙酮溶液, 合成了黄酮的
$\mathrm{C}(2) \sim \mathrm{C}(3)$ 环氧化产物, 产率 $90 \%$ 以上. 该环氧化产物 是热力学不稳定化合物, 尤其是 4'-位为供电子基团时, 在催化量的对甲苯磺酸作用下开环, 得到几乎定量的黄 酮醇(Scheme 15). 之后，在山柰酚和葪皮素衍生物的合 成中, 这种利用 DMDO 氧化黄酮为黄酩醇的方法, 因其 良好的产率而得到了广泛的应用 ${ }^{[35,36]}$.

2005 年, Hecht 及其合作者 ${ }^{[37]}$ 以柚皮素为原料，经 过查尔酮的 $\mathrm{I}_{2} / \mathrm{DMSO}$ 氧化环合, 黄酮的 DMDO 氧化, 高效地合成了山柰酚糖苷衍生物 SL0101，一种对核糖 体蛋白质激酶 p90 Rsk 具有显著抑制活性的天然产物, 探索了一条从黄烷酮到天然黄酮醇衍生物的便捷合成 途径(Scheme 16).

DMDO 本身不稳定，咜存时间短，且制备过程繁 琐，反应条件苛刻，在一定程度上制约了黄酮醇类化合 物的合成研究. 2009 年, Adams 等 ${ }^{[38]}$ 对 DMDO 环氧化反 应进行改进，将 DMDO 的制备和氧化反应合二为一，用 新鲜的 DMDO 原位反应制备黄酮醇. 后经吴峥等 ${ }^{[26]}$ 的 进一步改进，采用间歇式滴加过硫酸氢钾复合盐水溶液 的方法，对黄酮的共轭双键进行环氧化反应，合成黄酮 醇的产率达 $95 \%$ 以上. 改进后，既简化了合成 DMDO 的 步骤，又提高了黄酮醇的产率。该合成方法区域选择性 较好，几乎没有副产物生成，后处理相对简单，且整个 反应体系能在水溶液中进行, 不需要相转移催化剂, 反 应过程中没有有毒物质产生, 达到了当今绿色化学的基 本要求.<smiles></smiles>

图式 15 黄酩 DMDO 氧化法合成黄酮醇

Scheme 15 Synthesis of flavonols via flavones oxidation by DMDO 


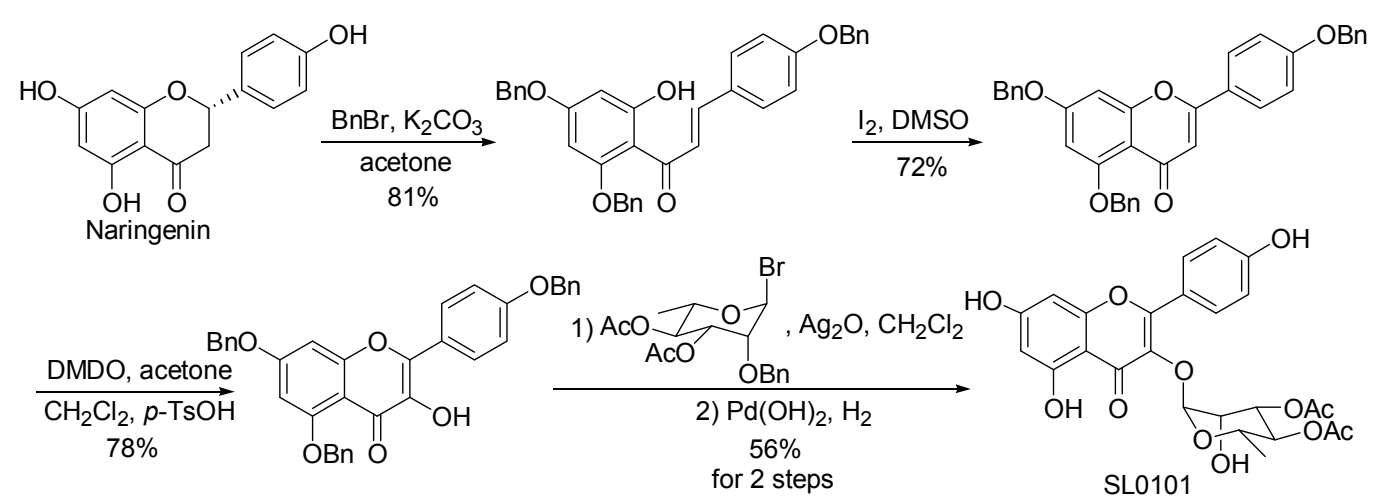

图式 16 山柰酚糖苷衍生物 SL0101 的合成路线

Scheme 16 Synthetic route of kaempferol glycoside SL0101

2011 年, Compton 等 ${ }^{[39]}$ 研究发现, 黄酮的 DMDO 氧 化受电子效应的影响显著. 当黄酮的 5-, 7-位被甲氧基 取代时，除了生成相应的黄酩醇外，羟基还会插入 $\mathrm{A}$ 环， 生成多个副产物(Scheme 17), 并且随着 DMDO 量的增 加各个副产物的量均会增加; 但是, 当 5-或 7-位的甲氧 基换为吸电子的乙酰氧基或苯甲酰氧基时, 主产物黄酮 醇的收率则显著增加.

另一种氧化黄酮的试剂是高价碘试剂: 碘苯二乙酯 $\left[\mathrm{PhI}(\mathrm{OAC})_{2}\right]$. 氧化得到的缩酮直接酸水解, 失去 3 分子 甲醇, 得到相应的黄酮醇, 总产率在 $60 \%$ 左 右 ${ }^{[40,41]}$ (Scheme 18).

利用 DMDO 等试剂氧化黄酮，制备黄酮醇，拓展了 黄酮醇的合成底物, 而且产率较高; 对于某些易于得到 的天然黄酮或黄烷酮原料, 不失为一种制备黄酮醇的简 易方法. 但是, 人们对 DMDO 等试剂所适用的黄酮底物 特征, 尚未得到规律性的认识, 尚需在合成实践中不断 探索.

\section{2 黄酮醇衍生物的合成}

黄酮醇衍生物的合成，是进一步研究这类化合物的<smiles>COc1cc(OC)c2c(OC)cc(=O)oc2c1</smiles>

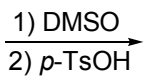<smiles>COc1cc(OC)c2c(=O)c(O)c(-c3ccccc3)oc2c1</smiles>

图式 17 5,7-二甲氧基黄酮的 DMDO 氧化

Scheme 17 Reaction of 5,7-dimethoxyflavone with DMDO<smiles>O=c1cc(-c2ccccc2)oc2ccccc12</smiles>

$\frac{\mathrm{Phl}(\mathrm{OAc})_{2}}{\mathrm{KOH}, \mathrm{MeOH}}$<smiles>CO[C@@]1(O)Oc2ccccc2C(O)Oc2ccccc21</smiles>
$\mathrm{HCl}$, acetone<smiles>O=c1c(O)c(-c2ccccc2)oc2ccccc12</smiles>

图式 18 黄酮碘苯二乙酯氧化法合成黄酮醇

Scheme 18 Synthesis of flavonols via flavones oxidation by iodobenzene diacetate 
Bouktaib 等 ${ }^{[48]}$ 采用连续的保护和脱保护, 实现了槲皮素 5 个酚羟基的单甲基化, 酚羟基表现出相对活性：7> $3 \approx 4^{\prime}>>5$; 然而, 在对二苯甲缩酮保护的槲皮素茮基 化时, 3-位苄基化产物为主产物(Scheme 19), 显示相对 活性：3>7. 邻二羟基二苯甲缩酮的存在, 使得 7-和 3羟基活性顺序发生反转. 这一实验结论也被后来一些研 究者所证实 ${ }^{[49,50]}$.

2010 年，何玲等 ${ }^{[51]}$ 进一步探索了䚞皮素的单甲基 化. 在微波辅助和相转移催化剂作用下, 利用嗍络合物 作保护基, 实现了槲皮素的区域选择性苄基化, 进而高 产率地合成了 4 个单甲基化衍生物(Scheme 20). 该方法 所选用的保护和脱保护试剂灵活多样, 且操作方便, 缩 短了反应时间，产率也有所提高，值得进一步研究.

综合上述文献, 黄酮醇酚羟基的相对活性大致表现 为: $7>4^{\prime} \approx 3>3^{\prime}>>5$. 5 -羟基与羰基 $(\mathrm{C}=\mathrm{O})$ 的氢键作用
致使其活性最弱，利用这一点，通过苄基等保护其它酚 着基，易于实现甲基化; 而 3-和 3'-差基活性与 4'-位差异 不大，同时由于空间因素的影响，甲基化较难实现. 但 是, 通过灵活运用保护和脱保护手段, 实现黄酮醇各个 酚羟基的区域选择性甲基化，大都能获得满意的产率. 然而，向某些天然黄酮醇的 $\mathrm{A}$ 环直接导入甲氧基等基 才，却一直困扰着人们. 2010 年, Lewin 科研小组 ${ }^{[19]}$ 报道 了一种通过卤原子间接导入甲氧基的方法. 合成路线设 计巧妙, 先用 NBS 在黄酮醇 A 环的 6-位和 8-位引入溴 原子, 再经碘甲烷在 3,7-位导入甲氧基, 最后在 $\mathrm{CuBr}$ 催 化下，发生溴甲氧基化亲核取代反应. 以橙皮苷为原料, 5 步反应合成了 $5,3^{\prime}$-二羟基-3,6,7,8,4'-五甲氧基黄酮(1 种具有显著的抗有丝分裂细胞毒活性的天然产物) (Scheme 21). 但是, 最后一步产物较为复杂，8-位甲氧 基化较困难，产率仅为 $20 \%$.<smiles>COc1cc(O)c2c(=O)c(O)c(-c3ccc(O)c(O)c3)oc2c1</smiles>

图式 19 槲皮素的 7-O-甲基化

Scheme 19 7-O-Methylation of quercetin

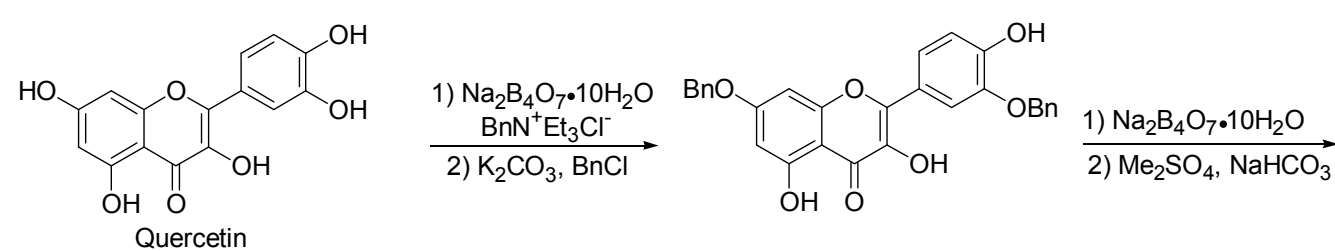<smiles>COc1ccc(-c2oc3cc(OCc4ccccc4)cc(O)c3c(=O)c2O)cc1OCc1ccccc1</smiles><smiles>COc1ccc(-c2oc3cc(O)cc(O)c3c(=O)c2O)cc1O</smiles><smiles>CC(=O)Oc1cc(OC(C)=O)c2c(=O)c(OC(C)=O)c(-c3ccc(OC(C)=O)c(OC(C)=O)c3)oc2c1</smiles><smiles>CC(=O)Oc1cc(-c2oc3cc(OCc4ccccc4)cc(OC(C)=O)c3c(=O)c2OC(C)=O)ccc1OCc1ccccc1</smiles>

1) $\mathrm{NaHCO}_{3}, \mathrm{BnN}^{+} \mathrm{Et}_{3} \mathrm{Cl}^{-}$

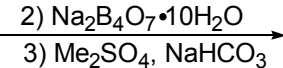<smiles>COc1cc(-c2oc3cc(OCc4ccccc4)cc(O)c3c(=O)c2O)ccc1OCc1ccccc1</smiles><smiles>COc1cc(-c2oc3cc(O)cc(O)c3c(=O)c2O)ccc1O</smiles>

图式 20 何小组檞皮素的选择性单甲基化

Scheme 20 Selective monomethylation of quercetin used by $\mathrm{He}$ 


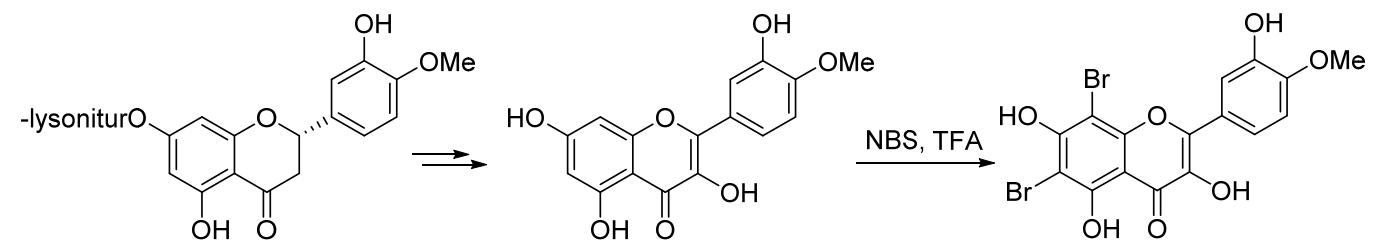<smiles>COc1ccc(-c2cc(-c3oc4c(OC)c(OC)c(OC)c(O)c4c(=O)c3OC)ccc2OC)cc1OC</smiles>

图式 21 由橙皮苷半合成多甲氧基黄酮

Scheme 21 Semisynthesis of polymethoxyflavone from Hesperidin

\section{2 黄酮醇的异戊烯基化}

异戊烯基化在芳香天然产物特别是黄酮、异黄酮、 香豆素、葱醌的结构多样性中扮演重要的角色 ${ }^{[52]}$. 目前 报道的异戊烯基化黄酮超过 1000 个. 从药用植物中发 现的许多异戊烯基化黄酮具有更为广泛、更强的生理活 性, 如抗肿瘤、抗雄激素、抗利什曼虫、抗 NO 的产生 等 ${ }^{[53]}$. 不同存在形式的异戊烯基侧链, 可使黄酮类化合 物的亲脂性得到提高, 有助于其在生物体中透过细胞 膜, 通过增加与生物膜的结合能力、改善与蛋白的作用, 而导致黄酮显著的生理活性改变 ${ }^{[54]}$. Pinostrobin 对 cAMP 磷酸二酯酶的抑制活性仅为 8-prenylpinostrobin 的 $1 / 6^{[55]}$, 其细胞毒活性为后者的 $1 / 3$ 至 $1 / 4^{[56]}$. 8 -位具有 异戊烯基、法呢烯基、 lavandylyl 的黄酮在多药耐药 [mutidrug resistance protein 1 (MRP1)/ATP-binding cassette $\mathrm{C} 1(\mathrm{ABCC} 1)$ inhibitor] 中更具有潜力 ${ }^{[57]}$. 增加异戊 烯基更是提高黄酮类化合物细胞毒活性的结构要求 ${ }^{[58]}$.

在黄酮醇母核上引入异戊烯基的早期报道，见于 Jain 等 ${ }^{[59]}$ 对山柰酚衍生物的合成. 他们用 2-甲基-3-丁
烯-2-醇试剂, 在 $\mathrm{BF}_{3} \cdot \mathrm{Et}_{2} \mathrm{O}$ 作用下, 与部分甲基化的山柰 酚偶联, 得到的产物是 6-和 8-异戊烯基以及 6,8-二异戊 烯基衍生物的混合物, 再利用柱层析分离纯化(Scheme 22). 这种异戊烯基直接连接到黄酮醇母核上的方法, 差基不需要保护, 反应一步进行, 操作简单; 但是, 原 料反应不完全, 产率低, 难以控制其位置选择性, 常常 得到单异戊烯基化和双异戊烯基化产物的混合物，难于 分离.

在黄酮结构中引入异戊烯基的常用方法, 是利用 Claisen 重排反应一一连接到苯环的 $O$-位异戊烯基团, 在碱性条件下加热重排到酚羟基的邻位或对位，成为 $C$-位异戊烯基团. 2014 年, Nemoto 小组 ${ }^{[60]}$ 采用该法合成 了 5'-异戊烯基檞皮素. 他们用 2-甲基 3-丁烯-2-基碳酸 异丁基酯试剂, 先将 2-甲基-3-丁烯-2-基引入到部分保 护的檞皮素的 4'-羟基上, 然后在乙酸酐作用下发生 Claisen 重排, 得到邻位重排产物, 产率为 $95 \%$. 脱去保 护基后，得到 5'-异戊烯基檞皮素(Scheme 23).<smiles>C=CC(C)(C)O</smiles><smiles>COc1ccc(-c2oc3c(CC=C(C)C)c(O)cc(O)c3c(=O)c2OC)cc1</smiles><smiles>COc1ccc(-c2oc3cc(O)c(CC=C(C)C)c(O)c3c(=O)c2OC)cc1</smiles><smiles>COc1ccc(-c2oc3c(CC=C(C)C)c(O)c(CC=C(C)C)c(O)c3c(=O)c2OC)cc1</smiles>

图式 22 黄酩醇类化合物的直接异戊烯基化

Scheme 22 Direct prenylation of flavonols 
<smiles>COc1cc(OC)c2c(=O)c(OC(C)=O)c(-c3ccc(O)c(OC(C)=O)c3)oc2c1</smiles>

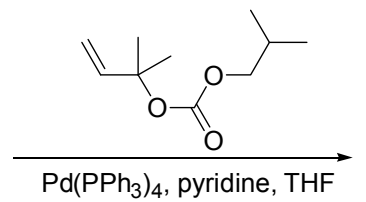<smiles></smiles><smiles>COc1cc(OC(C)=O)c2c(=O)c(OC(C)=O)c(-c3cc(CC=C(C)C)c(OC(C)=O)c(OC(C)=O)c3)oc2c1</smiles>

图式 23 Claisen 重排反应合成 5'-异戊烯基葪皮素

Scheme 23 Synthesis of uralenol via Claisen rearrangement

2005 年, Daskiewicz 等 ${ }^{[61]}$ 用异戊烯基溴试剂, 先将 异戊烯基引入到槲皮素的 5-羟基上, 再加热发生 Claisen 重排, 得到对位重排产物 8-异戊烯基槲皮素, 产 率为 $50 \%$ (Scheme 24).

为了提高 Claisen 对位重排反应的收率, Al-Maharik 等 ${ }^{[62]}$ 在合成天然产物 lupiwighteone 时, 首次选用 $\beta$-双酮 类稀土金属铕配合物 $\mathrm{Eu}(\mathrm{fod})_{3}[$ 三 $(6,6,7,7,8,8,8$-七氟-2,2二甲基-3,5-辛二酮)铕], 作为 Claisen 重排反应的催化 剂, 对位重排的产率达到 68\% (Scheme 25).

2013 年, 我们课题组 ${ }^{[63]}$ 在合成脱水淫羊蕉素时, 对
铕催化剂 $\mathrm{Eu}(\mathrm{fod})_{3}$ 促进的 Claisen 对位重排反应与高温 重排反应进行了比较(Scheme 26). 使用铕催化剂, 在氯 苯中 $80{ }^{\circ} \mathrm{C}$ 反应过夜，异戊烯基重排到 8-位的收率为 $42 \%$; 而在 $N, N$-二乙基苯胺中加热到 $217{ }^{\circ} \mathrm{C}$, 反应 $3 \mathrm{~h}$, 收率不到 $30 \%$. 这种使用铕催化剂的策略提高了对位重 排的选择性，而且大大降低了反应温度，从而抑制了部 分副反应的发生, 使得后处理相对简单化. 但是, 稀土 金属价格昂贵，而且具有毒性和不稳定性等问题，应用 受到一定限制.

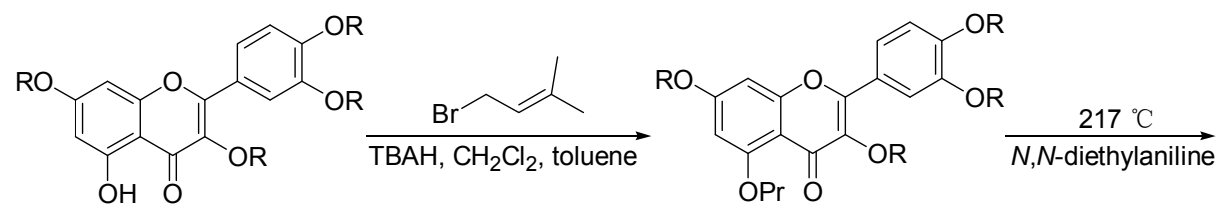<smiles>[R]Oc1ccc(-c2oc3c(CCC)c([R20])cc(O)c3c(=O)c2O[R])cc1O[R]</smiles><smiles>OCCO</smiles><smiles>CCCc1c(O)cc(O)c2c(=O)c(O)c(-c3ccc(O)c(O)c3)oc12</smiles><smiles>[R][R]([R])=[P-]CC=C(C)C</smiles>

图式 24 Claisen 对位重排反应合成 8-异戊烯基槲皮素

Scheme 24 Synthesis of 8-prenylated quercetin via para-Claisen rearrangement<smiles>CCOc1ccc(-c2coc3cc(OC(C)=O)cc(OCC=C(C)C)c3c2=O)cc1</smiles>

图式 $25 \mathrm{Eu}(\mathrm{fod})_{3}$ 催化的 Claisen 对位重排反应合成 lupiwighteone

Scheme 25 Synthesis of lupiwighteone via para-Claisen rearrangement catalyzed by $\mathrm{Eu}(\mathrm{fod})_{3}$ 


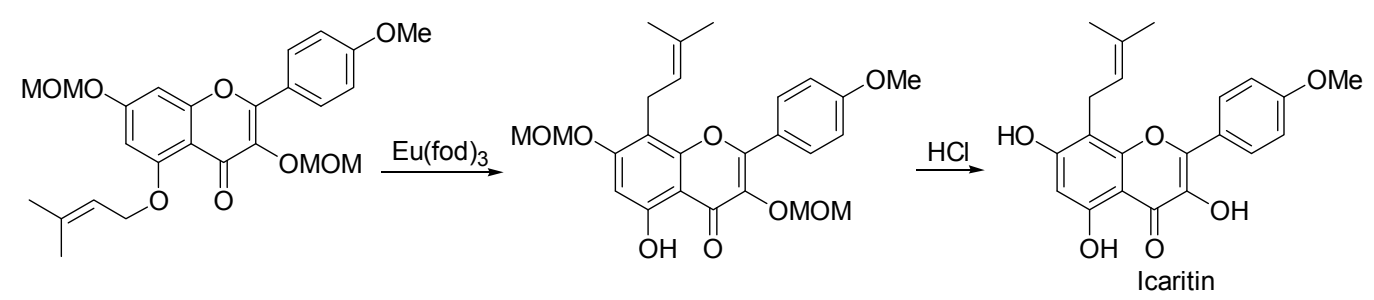

图式 26 脱水淫羊蕉素的合成路线

Scheme 26 Synthetic route of icaritin

此外, 异戊烯基的引入, 文献报道的方法还有: 碱 催化的异戊烯基溴与苯酚的反应 ${ }^{[64]}$ 、异戊烯基溴与芳基 锂试剂的反应 ${ }^{[65]}$ 、异戊烯基溴与芳香硼酸化合物或芳香 三氟硼酸钾盐的 Suzuki-Miyaura 反应 ${ }^{[6]}$ 、铱催化的二甲 基丙二烯与芳香底物的偶联反应 ${ }^{[67]}$ 、烯丙基醇与苯酚的 Mitsunobu 反应-Claisen 重排-烯烃复分解串联反应 ${ }^{[56]}$ 等. 但是, 这些方法往往选用催化剂较贵, 实验操作复杂, 副产物多并且难于分离. 本课题组正致力于黄酮类化合 物的异戊烯基化研究, 期望探索一种成本低廉、操作简 单、收率良好的具有普遍意义的异戊烯基引入方法.

\section{3 黄酮醇的糖苷化}

在天然黄酮苷中, 以槲皮素和山柰酚为代表的黄酮 醇类化合物约占 $80 \%$, 是许多药用植物的有效成分, 具 有抗氧化、抗炎抗真菌、抗 HIV-1、抗 MRSA 以及预防 过敏、保肝、雌性激素样作用等多种生理活性 ${ }^{[68 ~ 71]}$. 当 前黄酮苷类化合物主要从植物中提取分离, 而植物中的 含量又很低, 提取成本很高, 这无疑限制了其开发应用. 因此, 黄酮糖苷的合成, 始终是人们关注的热点问题.

黄酮糖苷合成常用的方法有 Koenigs-Knorr 法 ${ }^{[31,37]}$ 、 三氯乙酰亚胺酯法 ${ }^{[72]}$ 、相转移催化法 ${ }^{[73]}$. Koenigs-Knorr 法, 简单、成熟, 但是使用有毒、易爆并且价格昂贵的 银盐或录盐作催化剂, 反应条件苛刻, 后处理也较为繁 琐; 而且方法受底物与催化剂等的影响明显, 应用于黄 酮醇苷的合成产率较低.

Schmidt 三氯乙酰亚胺酯法, 糖供体稳定性好, 易 于保存, 反应立体选择性好. 该方法在寡糖和糖苷的合 成中应用广泛, 收率较高, 但应用于黄酮醇苷的合成中, 收率仍然不理想.

相转移催化法, 通常以溴代乙酰化糖作糖供体, 丙
酮、氯仿或 DMF 为溶剂, 在碱性条件下 (通常为 $\mathrm{KOH}$ 或 $\mathrm{K}_{2} \mathrm{CO}_{3}$ ), 通过相转移催化剂(通常为 TMEA 或 TBAB) 作用生成糖苷. 该方法反应体系简单、操作简便、条件 温和、立体选择性好, 通过不断改进, 收率得到明显提 高, 是黄酩醇糖苷化最常用的方法. $\mathrm{Li}$ 等 ${ }^{\left[{ }^{74]}\right.}$ 利用 $\mathrm{TBAB}$ 作相转移催化剂, 在黄酮醇的 3-位分别引入 4 种不同的 糖基(葡萄糖、木糖、半乳糖和阿拉伯糖), 合成了 16 个 糖苷(Scheme 27), 产率都在 50\%左右.

在对山柰酚和槲皮素的糖苷化中, $\mathrm{Yu}^{[75,76]}$ 和 $\mathrm{Du}$ 等 ${ }^{[77]}$ 课题组发现, 3-羟基糖苷化的适宜方法是相转移催 化法. 最佳反应条件是：在 $\mathrm{CHCl}_{3} / \mathrm{aq} . \mathrm{K}_{2} \mathrm{CO}_{3}(0.15$ $\left.\mathrm{mol} \cdot \mathrm{L}^{-1}\right)(V: V=1: 1)$ 体系中, 以溴代乙酰化糖作糖供 体, TBAB 为相转移催化剂, $50{ }^{\circ} \mathrm{C}$ 反应 $8 \mathrm{~h}$, 收率较高 (50\% 91\%). 相反, 对于 $3^{\prime}-$-, 4'-和 7-羟基的糖苷化, 适 宜使用糖基三氯乙酰亚胺酯法, $\mathrm{CH}_{2} \mathrm{Cl}_{2}$ 作溶剂, $\mathrm{AgOTf}$ 为催化剂, 可达到中等收率 $(52 \%)$.

2006 年, Needs 等 ${ }^{[78]}$ 在合成檞皮素的 3-和 $3 '-O$-葡萄 糖酸时, 进一步指出: 溴代乙酰化糖和糖基三氯乙酰亚 胺酯, 这两种不同的糖供体的区域选择性不同(Scheme 28). 以溴代乙酰化糖作供体, $\mathrm{Ag}_{2} \mathrm{O}$ 作催化剂, $\mathrm{CaSO}_{4}$ 为 脱水剂, 在 $0{ }^{\circ} \mathrm{C}$ 时于吡啶中反应 $16 \mathrm{~h}$, 只得到 3-位糖基 化产物, 总产率为 $40 \%$; 相反, 以糖基三氯乙酰亚胺酯 作供体, $\mathrm{BF}_{3} \cdot \mathrm{Et}_{2} \mathrm{O}$ 作催化剂, $3 \AA$ 分子篮为脱水剂, 在 $\mathrm{CH}_{2} \mathrm{Cl}_{2}$ 中室温反应过夜, 只得到 3'-位糖基化产物, 总产 率为 $11 \%$.

在黄酮醇 3-羟基的糖苷化中，苷元上其余羟基的保 护和脱保护常常比较繁琐; 同时, 3-羟基的氢键作用, 导致糖苷化的产率不高. 为了克服这两个致命弱点, Oyama 等 ${ }^{[79}$ 将糖基的引入置于黄酮醇骨架的构建之前,
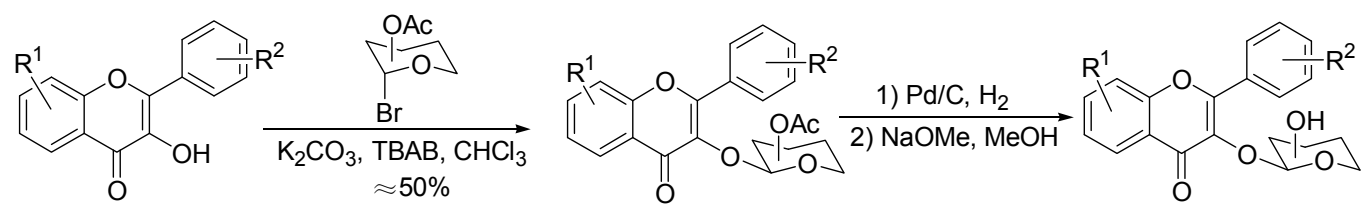

图式 $27 \mathrm{Li}$ 小组黄酮醇 3-O-糖苷的合成路线

Scheme 27 Synthetic route of 3-O-glycosylated flavonols used by $\mathrm{Li}$ 


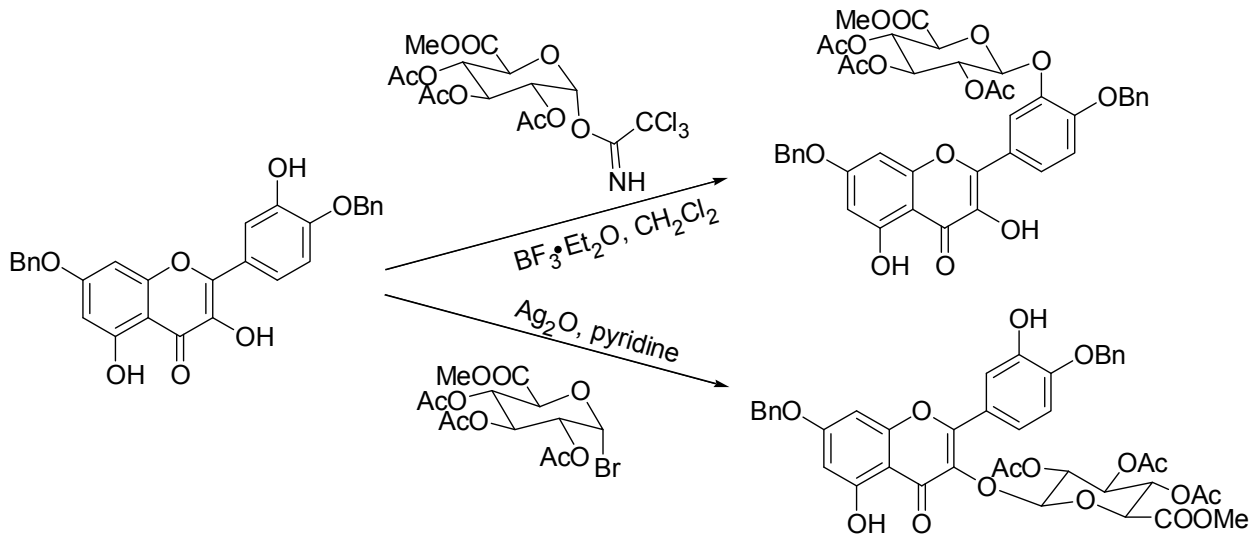

图式 28 Needs 小组檞皮素 3-和 3'- $O$-糖苷衍生物的合成路线

Scheme 28 Synthetic route of quercetin 3-and 3'-O-glycosylated derivatives used by Needs

将保护反应和糖基化反应合二为一, 缩短了反应步骤, 总产率有所提高(Scheme 29). 但是, 这种合成思路也有 一定的局限性. 首先, 在向酮羰基的 $\beta$-位引入糖基时, 要用苄基保护的糖基三氯乙酰亚胺酯, 不能使用乙酰基 保护的糖供体, 可能是因为乙酰基易于水解, 从而使糖 供体失去活性; 而且, 引入糖基时伴随着硅烷基的部分 脱落, 需要再次上保护基. 其次, 在随后的 BakerVekataraman 反应与脱水环合步骤中, 需要较高的温度, 产率也不高. 这些因素都制约着该方法的推广应用.

黄酮醇糖苷化的文献报道较多, 但总体产率不高, 步骤繁多且分离复杂, 其化学合成面临诸多挑战, 而模 拟生物酶催化正在给这一领域注入新的活力.

黄酮醇的衍生化，除了上述 3 类主要的衍生化反应, 此外还有: Sagrera 等 ${ }^{[4]}$ 报道的双黄酮醇衍生物的合成, Porco 小组 ${ }^{[80]}$ 发展的黄酮醇与桂皮酸酯的 $[3+2]$ 光环化 加成反应, 合成具有环戊醇并苯并呋喃骨架的潜在抗癌 药物洛克米兰酸酯类化合物等. 这些结构新颖、性质独 特的衍生物的合成, 丰富了黄酮醇大家族, 推动了对新
型合成方法的研究，更为新药的发展提供了新的思路和 方向.

\section{3 结束语}

黄酮醇类化合物独特而重要的生物和药理活性, 使 得其合成方法研究一直备受有机化学和药物化学家们 的重视. 由于黄酮醇类化合物结构复杂、反应位点多, 区域选择性往往难于控制，常常需要多步的保护和脱保 护; 同时, 其固有的特性如溶解性差、对酸碱性敏感等, 使得分离和纯化处理亦较为繁杂，因此，其合成和修饰 的产率普遍不高. 这些都是对黄酮醇类化合物进行深入 研究所面临的极具挑战性的课题. 但是, 随着有机合成 新方法和新技术的不断涌现，研究的不断深入，相信在 不久的将来会出现更加有效、更加经济和环保的合成黄 酮醇的方法. 本文旨在能够对从事黄酮醇类化合物合成 研究的工作者们有所裨益.<smiles>CC(C)(C)Oc1cc(O)c(C(=O)CO)c(O[SbH3])c1</smiles>

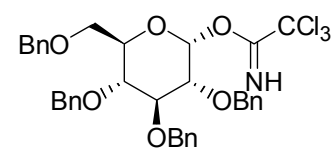
1) TMSOTf, MS $4 \stackrel{\circ}{\AA} \mathrm{MeCN}$ 2) $\mathrm{TBSCl}, \mathrm{Et}_{3} \mathrm{~N}, \mathrm{CH}_{2} \mathrm{Cl}_{2}$ $57 \%$

TBSO

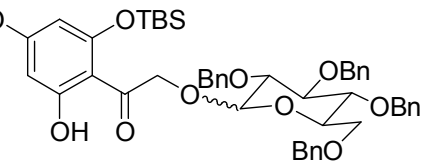

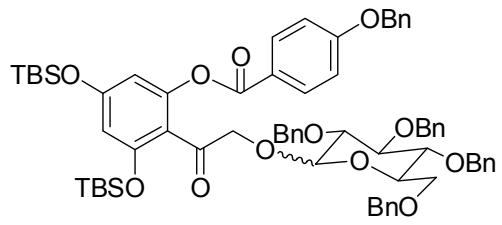

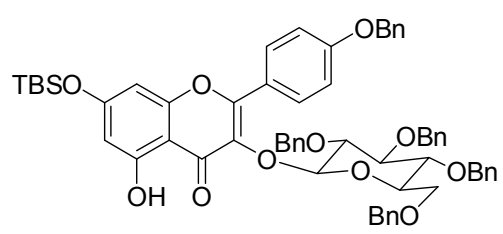

图式 29 Oyama 小组檞皮素 3-O-糖苷衍生物的合成路线

Scheme 29 Synthetic route of quercetin 3-O-glycosylated derivatives used by Oyama 


\section{References}

[1] Amado, N. G.; Fonseca, B. F.; Cerqueira, D. M.; Neto, V. M.; Abreu, J. G. Life Sci. 2011, 89(15 16), 545.

[2] Santos, M. C. S.; Goncalves, C. F. L.; Vaisman, M.; Ferreira, A. C. F.; Carvalho, D. P. Food Chem. Toxicol. 2011, 49(10), 2495.

[3] Yang, L. J.; Lu, D. F.; Guo, J. J.; Meng, X. L.; Zhang, G. L.; Wang, F. J. Ethnopharmacol. 2013, 145(3), 715.

[4] Huxley, R. R.; Neil, H. Eur. J. Clin. Nutr. 2003, 57, 904.

[5] Miller, K. D.; Strommer, J.; Taylor, L. P. Plant Mol. Biol. 2002, 48(3), 233.

[6] Klymchenko, A. S.; Ozturk, T.; Demchenko, A. P. Tetrahedron Lett. 2002, 43(39), 7079.

[7] Xu, S. J.; Shao, Y.; Ma, K.; Cui, Q. H.; Liu, G. Y.; Wu, F.; Li, M. J. Analyst 2011, 136(21), 4480.

[8] Zamotaiev, O. M.; Postupalenko, V. Y.; Shvadchak, V. V.; Pivovarenko, V. G.; Klymchenko, A. S.; Mely, Y. Bioconjugate Chem. 2011, 22(1), 101.

[9] Chen, D. Y.; Chen, C. L.; Cheng, Y. M.; Lai, C. H.; Yu, J. Y.; Chen, B. S.; Hsieh, C. C.; Chen, H. C.; Chen, L. Y.; Wei, C. Y.; Wu, C. C.; Chou, P. T. ACS Appl. Mater. Interfaces 2010, 2(6), 1621.

[10] Auwers, K.; Muller, K. Ber. Dtsch. Chem. Ges. 1908, 41, 4233.

[11] Bird, C. W.; Cookson, R. C. J. Org. Chem. 1959, 24(3), 441.

[12] Wang, Z. R. Comprehensive Organic Name Reactions and Reagents, John Wiley \& Sons, Inc. 2010, pp. 52 $\sim 56$.

[13] Wang, Q. A.; Fang, W. Q.; Liao, T. G. J. Hunan Univ. (Nat. Sci. ) 2006, 33(6), 98 (in Chinese).

(汪秋安, 方伟琴, 廖头根, 湖南大学学报(自然科学版), 2006, 33(6), 98.)

[14] Yap, S.; Loft, K. J.; Woodman, O. L.; Williams, S. J. ChemMedChem 2008, 3, 1572.

[15] Black, D. S.; Kumar, N.; Mitchell, P. S. R. J. Org. Chem. 2002, 67(8), 2464

[16] Rodriguez, E.; Carman, N. J.; Vander Velde, G.; McReynolds, J. H.; Mabry, T. J.; Irwin, M. A.; Geissman, T. A. Phytochemistry 1972, $11(12), 3509$.

[17] Stermitz, F. R.; Scriven, L. N.; Tegos, G.; Lewis, K. Planta Med. 2002, 68(12), 1140.

[18] Kraus, G.; Roy, S. J. Nat. Prod. 2008, 71, 1961.

[19] Lewin, G.; Maciuk, A.; Thoret, S.; Aubert, G.; Dubois, J.; Cresteil, T. J. Nat. Prod. 2010, 73(4), 702.

[20] Pandurangan, N.; Bose, C.; Banerji, A. Bioorg. Med. Chem. Lett. 2011, 2l(18), 5328.

[21] Gunduz, S.; Goren, A. C.; Ozturk, T. Org. Lett. 2012, 14(6), 1576.

[22] Bennett, M.; Burke, A. J.; O'Sullivan, W. I. Tetrahedron 1996, 52(20), 7163 .

[23] Stevens, J. F.; Miranda, C. L.; Frei, B.; Buhler, D. R. Chem. Res. Toxicol. 2003, 16(10), 1277.

[24] Bhattacharyya, S.; Hatua, K. RSC Adv. 2014, 4(16), 18702.

[25] Geissman, T. A.; Fukushima, D. K. J. Am. Chem. Soc. 1948, 70(5), 1686.

[26] Wu, Z.; Cai, S. L.; Fan, W. J.; Wang, Q. A. Chin. J. Org. Chem. 2012, 32, 1296 (in Chinese). (吴峥，蔡双莲，范文金，汪秋安，有机化学, 2012, 32, 1296.)

[27] Bois, F.; Beney, C.; Mariotte, A. M.; Boumendjel, A. Synlett 1999, 9, 1480.

[28] Ganguly, A. K.; Kaur, S.; Mahata, P. K.; Biswas, D.; Pramanik, B. N.; Chan, T. M. Tetrahedron Lett. 2005, 46, 4119.

[29] Fougerousse, A.; Gonzalez, E.; Brouillard, R. J. Org. Chem. 2000, 65(2), 583.

[30] Tanaka, H.; Stohlmeyer, M. M.; Wandless, T. J.; Taylor, L. P. Tetrahedron Lett. 2000, 41(50), 9735.
[31] Urgaonkar, S.; Shaw, J. T. J. Org. Chem. 2007, 72(12), 4582.

[32] Chen, L. L.; Li, J.; Luo, C.; Liu, H.; Xu, W. J.; Chen, G.; Liew, O. W.; Zhu, W. L.; Puah, C. M.; Shen, X.; Jiang, H. L. Bioorg. Med. Chem. 2006, 14(24), 8295.

[33] Costa, A. M.; Dean, F. M.; Jones, M. A.; Varma, R. S. J. Chem. Soc., Perkin Trans. 1 1985, 799.

[34] Adam, W.; Golsch, D.; Hadjiarapolou, L.; Patonay, T. J. Org. Chem. 1991, 56(26), 7292.

[35] Lee, Y. J.; Wu, T. D. J. Chin. Chem. Soc. 2001, 48(2), 201.

[36] Chu, H. W.; Wu, H. T.; Lee, Y. J. Tetrahedron 2004, 60(11), 2647.

[37] Maloney, D. J.; Hecht, S. M. Org. Lett. 2005, 7(6), 1097.

[38] Adams, T. E.; Sous, M. E.; Hawkins, B. C.; Hirner, S.; Holloway, G.; Khoo, M. L.; Owen, D. J.; Savage, G. P.; Scammells, P. J.; Rizzacasa, M. A. J. Am. Chem. Soc. 2009, 131(4), 1607.

[39] Compton, B. J.; Larsen, L.; Weavers, R. T. Tetrahedron 2011, 67(4), 718.

[40] Moriarty, R. M.; Prakash, O.; Musallam, H. A. J. Heterocycl. Chem. 1985, $22(2), 583$.

[41] Sagrera, G.; Seoane, G. Synthesis 2010, 2776.

[42] Beutler, J. A.; Hamel, E.; Vlietinck, A. J.; Haemers, A.; Rajan, P.; Roitman, J. N.; Cardellina II, J. H.; Boyd, M. R. J. Med. Chem. 1998, 4l(13), 2333.

[43] Lopez-Lazaro, M.; Galvez, M.; Martin-Cordero, C.; Ayuso, M. J. Stud. Nat. Prod. Chem. 2002, 27, 891.

[44] Li, W. X.; Cui, C. B.; Cai, B.; Wang, H. Y.; Yao, X. S. J. Asian Nat. Prod. Res. 2005, 7(4), 615.

[45] Jurd, L. J. Am. Chem. Soc. 1958, 80, 5531.

[46] Jurd, L. J. Org. Chem. 1962, 27, 1294.

[47] Rao, K. V.; Owoyale, J. A. J. Heterocycl. Chem. 1976, 13(6), 1293.

[48] Bouktaib, M.; Lebrun, S.; Atmani, A.; Rolando, C. Tetrahedron 2002, 58(50), 10001.

[49] Chen, Z. W.; Hu, Y. Z.; Wu, H. H.; Jiang, H. D. Bioorg. Med. Chem. Lett. 2004, 14 (15), 3949.

[50] Li, N. G.; Shi, Z. H.; Tang, Y. P.; Yang, J. P.; Lu, T. L.; Zhang, F.; Huang, Y. W.; Wang, Z. J.; Duan, J. A. Chin. Chem. Lett. 2011, 22(1), 5 .

[51] Zhou, Z. H.; Fang, Z.; Jin, H.; Chen, Y.; He, L. Synthesis 2010, 3980.

[52] Yazaki, K.; Sasaki, K.; Tsurumaru, Y. Phytochemistry 2009, 70, 1739.

[53] Alhassan, A. M.; Abdullahi, M. I.; Uba, A.; Umar, A. Trop. J. Pharm. Res. 2014, 13(2), 307.

[54] Clark, M. K.; Scott, S. A.; Wojtkowiak, J.; Chirco, R.; Mathieu, P.; Reiners, J. J.; Mattingly, R. R.; Borch, R. F.; Gibbs, R. A. J. Med. Chem. 2007, 50(14), 3274.

[55] Nikaido, T.; Ohmoto, T.; Kinoshita, T.; Sankawa, U.; Monache, F. D.; Botta, B.; Tomimori, T.; Miyaichi, Y.; Shirataki, Y.; Yokoe, I.; Komatsu, M. Chem. Pharm. Bull. 1989, 37(5), 1392.

[56] Poerwono, H.; Sasaki, S.; Hattori, Y.; Higashiyama, K. Bioorg. Med. Chem. Lett. 2010, 20(7), 2086.

[57] Bobrowska-Hagerstrand, M.; Wrobel, A.; Mrowczynska, L.; Soderstrom, T.; Shirataki, Y.; Motohashi, N.; Molnar, J.; Michalak, K.; Hagerstrand, H. Oncol. Res. 2003, 13, 463.

[58] Li, F.; Awale, S.; Tezuka, Y.; Kadota, S. Biol. Pharm. Bull. 2009, 32(12), 2075.

[59] Jain, A. C.; Gupta, R. K. Aust. J. Chem. 1975, $28(3), 607$.

[60] Kawamura, T.; Hayashi, M.; Mukai, R.; Terao, J.; Nemoto, H. Synthesis 2014, 46(2), 170.

[61] Daskiewicz, J. B.; Depeint, F.; Viornery, L.; Bayet, C.; Comte-Sarrazin, G.; Comte, G.; Gee, G. M.; Johnson, I. T.; Ndjoko, K.; Hostettmann, K.; Barron, D. J. Med. Chem. 2005, 48(8), 2790.

[62] Al-Maharik, N.; Botting, N. P. Tetrahedron 2003, 59, 4177. 
[63] Mu, G. M.; Pu, W. C.; Zhou, M.; Liu, Y.; Yang, H. J.; Wang, C. Chin. J. Org. Chem. 2013, 33(6), 1298 (in Chinese).

(牟关敏, 蒲文臣, 周敏, 刘燕, 杨海君, 王淳, 有机化学, 2013, 33(6), 1298.)

[64] Yang, J. H.; Jiang, S. Z.; Zhao, Y. M.; Li, Y. F.; Ji, C. B.; Liu, W. Y. Chin. Chem. Lett. 2009, 20(9), 1062.

[65] Park, B. H.; Lee, H. J.; Lee, Y. R. J. Nat. Prod. 2011, 74(4), 644.

[66] Gerbino, D. C.; Mandolesi, S. D.; Schmalz, H. G.; Podesta, J. C. Eur. J. Org. Chem. 2009, 3964.

[67] Zhang, Y. J.; Skucas, E.; Krische, M. J. Org. Lett. 2009, 11(18), 4248.

[68] Ahn, M. J.; Kim, C. Y.; Lee, J. S.; Kim, T. G.; Kim, S. H.; Lee, C. K.; Lee, B. B.; Shin, C. G.; Huh, H.; Kim, J. Planta Med. 2002; 68(5), 457.

[69] Smith, J. A.; Maloney, D. J.; Hecht, S. M.; Lannigan, D. A. Bioorg. Med. Chem. 2007, 15(14), 5018.

[70] You, H. J.; Ahn, H. J.; Ji, G. E. J. Agric. Food Chem. 2010, 58(20), 10886.
[71] Veitch, N. C.; Grayer, R. J. Nat. Prod. Rep. 2011, 28, 1626.

[72] Caldwell, S. T.; Petersson, H. M.; Farrugia, L. J.; Mullen, W.; Crozier, A.; Hartley, R. C. Tetrahedron 2006, 62(31), 7257.

[73] Ren, X. H.; Shen, L. L.; Muraoka, O.; Cheng, M. S. J. Carbohydr. Chem. 2011, 30, 119.

[74] Li, Z. T.; Ngojeh, G.; DeWitt, P.; Zheng, Z.; Chen, M.; Lainhart, B.; Li, V.; Felpo, P. Tetrahedron Lett. 2008, 49, 7243.

[75] Li, M.; Han, X. W.; Yu, B. Tetrahedron Lett. 2002, 43, 9467.

[76] Yang, W. Z.; Sun, J. S.; Lu, W. X.; Li, Y.; Shan, L.; Han, W.; Zhang, W. D.; Yu, B. J. Org. Chem. 2010, 75(20), 6879.

[77] Du, Y. G.; Wei, G. H.; Linhardt, R. J. Tetrahedron Lett. 2003, 44, 6887.

[78] Needs, P. W.; Kroon, P. A. Tetrahedron 2006, 62, 6862.

[79] Oyama K.; Kawaguchi, S.; Yoshida, K.; Kondo, T. Tetrahedron Lett. 2007, 48, 6005.

[80] Roche, S. P.; Cencic, R.; Pelletier, J.; Porco Jr., J. A. Angew. Chem., Int. Ed. 2010, 49(37), 6533. 